"Essai de sociologie de la mémoire : le cas des camps annexes de Drancy dans Paris ", Genèses, dossier « Histoire et oubli », n61, décembre 2005, p. 47-69.

\title{
Essai de sociologie de la mémoire : le cas du souvenir des camps annexes de Drancy dans Paris
}

Sarah Gensburger

« Tabou » (Ternon 1999 ; Ferro 2002) et «syndrome » (Rousso 1990), d'un côté, « abus » (Todorov 1995 ; Ricœur 2000: 82-111) et «concurrence » (Chaumont 1997), de l'autre, l'analyse actuelle de la mémoire emprunte le plus souvent aux vocabulaires respectifs de la psychanalyse et de la guerre économique. Elle oscille ainsi entre la mise en avant d'une conscience collective, plus exactement d'un « inconscient », et la mise en évidence, souvent faite dénonciation, de quelques stratèges ${ }^{1}$. Au-delà de leur opposition, ces deux approches ont cependant en commun une prise en compte de la mémoire par son écume. Concentrées sur le pour quoi, elles négligent la question du comment (Gensburger et Lavabre 2005 : 76-95). Si leurs tenants emploient régulièrement le concept de "mémoire collective» emprunté à Maurice Halbwachs, ils semblent n'y voir qu'une forme réifiée, largement institutionnalisée, de l'évocation du passé (Confino : 1386-1403 ; Lavabre : 48-57). Pourtant, dans la suite des réflexions de cet auteur pionnier, enrichies de celles de Roger Bastide ${ }^{2}$, il est possible de prendre la mémoire au sérieux en faisant l'hypothèse qu'elle est, au moins en partie, un fait social. Une recherche empirique sur les camps annexes de Drancy dans Paris (Dreyfus et Gensburger 2003) fournit la matière de cette appréhension de l'objet mémoire. Comme dans les travaux de Michael Pollak (1990 ; 1993 : 15-39), notre démarche initiale d'histoire orale nous a conduite à une analyse sociologique du souvenir et de ses mécanismes (Raphaël 1980). Si le corpus de cette recherche historienne fut originellement constitué d'archives, publiques comme privées, et d'entretiens avec d'anciens internés ou certains de leurs descendants, nos réflexes de sociologue nous incitèrent cependant à l'enrichir progressivement de données a priori accessoires pour la restitution factuelle du passé mais nécessaires à la compréhension de leur(s) mémoire(s). Séances d'observation participante auprès d'associations et d'acteurs concernés, enquête topographique sur les lieux mêmes d'internement ou lecture de publications diverses vinrent s'adjoindre aux sources de départ. Le présent essai de sociologie de la mémoire repose donc sur un matériau diversifié. Centré sur la compréhension du comment, il entend essayer de combler, certes très partiellement, le fossé qui, dans le champ de l'étude de la «mémoire », sépare parfois les considérations théoriques de réelles bases empiriques. 
Jusqu'à très récemment, l'existence de ces camps de travail forcé était méconnue par les historiens $^{3}$, souvent inconnue par les autres. Les témoins potentiels étaient eux-mêmes, pour leur très grande majorité, restés mués. Longtemps, seules des bribes du passé ont perduré. Comblant le trou de mémoire qui entourait ces lieux d'internement, des images et scénarios empruntés aux formes structurées, diffusées et dominantes, des représentations du passé, telles que la spoliation artistique et la déportation raciale, s'étaient imposées. Elles persistent parfois encore aujourd'hui. Ce constat conduit à s'interroger sur les raisons de ce relatif silence et celles de son évolution. Leur explication passe par la mise en relation des processus de remémoration avec leurs conditions sociales de possibilité et de structuration. La mémoire apparaît comme un fait social dont les déterminants peuvent être mis en évidence. Si la complexité de l'identification sociale, notamment à l'égard du judaïsme, de la majorité des internés des camps annexes de Drancy dans Paris constitue une des principales spécificités de ces lieux, elle fournit également l'explication centrale de la faible remémoration qu'ils ont connue, au sens où celle-ci n'a pu être portée par des phénomènes collectifs.

\section{Histoire des camps annexes de Drancy dans Paris}

Les camps annexes de Drancy dans Paris se situent au croisement de la déportation physique et de la spoliation économique. Lorsque, entre décembre 1941 et fin janvier 1942 (Roseman 2002), la décision d'assassiner les Juifs d'Europe fut prise, Alfred Rosenberg dirigeait depuis déjà plus d'un an une administration responsable de la spoliation artistique. Nous ne savons pas avec certitude s'il était au courant de la mise en place de la Solution finale lorsque, le 18 décembre 1941, il envoya à Adolf Hitler une note demandant que ses services parisiens soient autorisés à vider les appartements que les Juifs n'habitaient plus. Il justifiait sa demande en expliquant que les biens manquaient dans les territoires de l'Est également placés sous son contrôle. Hitler notifia son accord le 31 décembre 1941. L’opération fut baptisée «Möbel Aktion » (opération meuble) (Azoulay et Wieviorka 2000). Le butin devait être distribué aux civils du Reich. Afin de mener cette opération, un nouveau service fut créé : la Dienststelle Westen, « Service Ouest », officiellement mise en place le 17 avril 1942, sous la direction de Kurt von Behr. Si les entreprises de déménagement françaises, réquisitionnées, vidaient les appartements, pour le reste, ce dernier ne disposait que d'un faible effectif principalement constitué de soldats mutilés. C'est ainsi que, en juillet 1943, la Dienststelle Westen se mit à louer de la main-d'œuvre au SD (Sicherheitsdienst, service de sécurité) de Drancy. Chef d'un service de spoliation, Kurt von Behr devint chef de camp. 
À Drancy, Aloïs Brunner attendait les arrivées massives censées suivre la rédaction du décret de dénaturalisation des Juifs devenus français après 1927, prévue pour l'été 1943. Soucieux de décharger l'effectif du camp, il loua à la Dienststelle Westen les internés classés comme temporairement « non-déportables ». En effet, à peine arrivé à la direction du camp de transit, et conformément à la classification mise en œuvre par le régime nazi, il avait classé les détenus. Les Juifs « conjoints d'aryens », les « demi-Juifs », ou « mischlinge », et les femmes juives de prisonniers de guerre juifs devaient rester internés dans l'Hexagone, comme otages potentiels pour ces dernières, sans statut défini pour les premiers car, en haut lieu, on ne parvenait pas à se mettre d'accord sur leur sort final (Stoltzfus 1996). Les trois catégories devaient en principe composer les effectifs transférés à Paris.

Le 18 juillet 1943, 180 internés quittèrent Drancy pour le magasin Lévitan du 85-87 rue du Faubourg-Saint-Martin dans le $10^{\mathrm{e}}$ arrondissement, aryanisé quelques jours auparavant. Le $1^{\mathrm{er}}$ novembre suivant, le second camp parisien, dit «Austerlitz», situé au 43 quai de la Gare ${ }^{4}$ dans le $13^{\mathrm{e}}$ arrondissement, reçut à son tour un groupe de près de 200 détenus. Dans ces deux lieux, les internés étaient soumis au travail forcé. Toute la journée, ils triaient, nettoyaient et réparaient objets et meubles pillés dans les appartements juifs. Le 15 mars 1944, un troisième camp, de taille plus réduite, vit le jour dans 1 le $16^{\mathrm{e}}$ arrondissement, au 2 rue Bassano, dans un hôtel particulier aryanisé, ayant appartenu aux Cahen d'Anvers (Assouline 1997). Les couturières y confectionnaient des robes, des costumes et uniformes pour les Allemands. Différents artisans y fabriquaient ou y remettaient en état une large gamme de biens de qualité. Dans ces trois lieux, les détenus ont assisté à toutes les étapes de l'opération meuble qui au 31 juillet 1944 avait vidé 69619 logements, chargé 674 trains et produit des valeurs pour un montant de 11695516 reichsmarks.

Cette affectation au travail dans la capitale signifiait pour les internés des conditions de vie meilleures que celles en vigueur à Drancy. La nourriture y était moins rare, le régime disciplinaire plus souple. En principe, demeurer dans les camps annexes signifiait surtout échapper au départ, fatal, vers l'Est. Pour une large majorité de leurs prisonniers, les camps parisiens ont ainsi constitué un abri temporaire qui, avec le rapatriement à Drancy, le 12 août 1944, puis la libération du camp six jours plus tard, se révéla permanent. Les détenus n’en restaient pas moins dépendants du camp principal. Caractéristique du fonctionnement administratif nazi, l'arbitraire était permanent. En cas d'évasion ou d'insoumission, plusieurs internés étaient ramenés au camp principal pour y être déportés. 


\section{Encadré 1 : «Identifier les internés : listes, registres, fichiers »}

Si le débat sur le « Fichier juif » a rappelé le rôle de l'enregistrement administratif (Conan et Rousso 1996 : 97-156) dans la déportation et l'extermination, les fiches ou autres listes qui en furent le support sont pourtant rarement présentes en l'état dans les archives. L'ampleur du travail accompli par Serge Klarsfeld pour réaliser son Mémorial en fournit une parfaite illustration. Il en va de même pour l'identification des anciens détenus des camps annexes de Drancy dans Paris. Les archives administratives de la Dienststelle Westen ayant été détruites à la Libération, si des registres spécifiques de ces lieux ont jamais existé, ils ont disparu. De même, le fichier individuel de Drancy ne précise que très exceptionnellement l'affectation de l'interné à Paris.

À l'inverse, à partir de septembre 1943, le registre des entrées et sorties du camp de transit indique presque systématiquement l'effectif global affecté à la Dienststelle Westen. Ce chiffre est également mentionné dans les documents des archives des cuisines de l'Union générale des Israélites de France (Centre de documentation juive contemporaine, UGIF 48, « Menus St Martin, Austerlitz, Bassano », 1943-1944) qui avait en charge la gestion logistique des camps. Si les deux types de données divergent à la marge, ils convergent quant à l'ordre de grandeur qu'ils donnent à voir et permettent de situer entre 690 et 850 le nombre des détenus passés par ces annexes. Aucun de ces documents ne fournit toutefois les noms et prénoms des personnes qui ont appartenu à cet ensemble.

Pourtant, reposant sur d'autres documents, le travail d'identification individuelle des internés aboutit à la même valeur d'ensemble. Notre élaboration d'un fichier nominatif comme outils de travail relève ainsi de plusieurs sources. Trois listes nominatives intermédiaires ont été utilisées. La plus complète, la première, constitue un état, par camp, des identités nominatives des internés, dressé à la fin du mois de janvier 1944 (CDJC, UGIF 92, 1679 à 1681, Liste « Austerlitz, Lévitan, Bassano », non datée). Établie aux alentours du $1^{\text {er }}$ novembre 1943, la deuxième énumère ceux et celles qui sont envoyés de Drancy à Paris pour l'ouverture du camp d'Austerlitz (CDJC, CCCLXXVI-4, Liste « Dienststelle Westen », 29 octobre 1943). La dernière est en réalité un ensemble composé des listes établies toutes les semaines par les chefs juifs des trois camps à l'attention des services de l'UGIF (CDJC, UGIF, par exempe 14, 1100, « Bordereau de linge envoyé à l'UGIF », 24 avril 1944). Entre juillet 1943 et août 1944, ces listes ont permis le fonctionnement de la blanchisserie. Pour laver leur linge, les internés devaient en effet remettre celui-ci aux services de l'UGIF qui les faisaient suivre, à 
l'extérieur, aux familles ou amis non-juifs restés libres qui se chargeaient de cette tâche. Cette identification nominative est complétée par la lecture intégrale du "Carnet de fouilles » de Drancy qui indiquait régulièrement si le nouvel entrant revenait de « Bassano », «Lévitan » ou « Austerlitz ».

Ces recoupements ont permis d'établir un registre de 795 personnes précisément identifiées. Cet outil de travail peut être désigné du titre de «Identités des internés qui ont séjourné dans l'un des camps annexes de Drancy dans Paris ». Il a rendu possible de déterminer ceux et celles d'entre eux qui ont été déportés. Pour cela, le document établi a été croisé avec les listes fournies par Serge Klarsfeld dans son Mémorial de la Déportation (Klarsfeld 1978).

795 personnes ont été identifiées comme ayant séjourné dans l'un des trois lieux entre juillet 1943 et août 1944. 20\% d'entre elles ont été déportées. Sur un total de 166 déportés, 64 le furent à Bergen-Belsen, 102 à Auschwitz. Les 80\% restant ont finalement été ramenés à Drancy pour être déportés par Aloïs Brunner qui comptait organiser un ultime grand convoi. Ce dernier n'eut finalement pas lieu, les détenus des camps annexes dans Paris ont été libérés à Drancy le 18 août 1944.

\section{Oubli et trou de mémoire}

Avec la libération de leurs internés, et malgré leur fort taux de non déportation, ces trois camps annexes de Drancy dans Paris n'ont pas donné lieu à des récits et témoignages. Sur l'explication de ce silence, les hypothèses sont multiples. Pour nombre de journalistes, il s'agit d'un tabou social voire d'une volonté d'étouffement politique ${ }^{5}$. Pour d'autres, ces lieux d'internement seraient tout simplement trop petits, sans réelle importance, ce qui ferait qu'on n'en ait pas parlé plus tôt. Après tout, comme l'a expliqué S. Klarsfeld à l'une des journalistes qui lui demandait son opinion à la sortie de l'ouvrage qui en restitue l'histoire (Dreyfus, Gensburger 2003) : «Ce ne fut pas une tragédie. La tragédie c'est que près de 80000 Juifs de France furent victimes de la déportation et que l'État français en fut complice» (The Jerusalem Report 2004).

Dire cela c'est notamment omettre, notamment, le rôle, certes limité, de déversoir du camp de transit de Drancy qu'ont joué ces camps. Mais la question n'est pas là. Avant tout, ces différentes «raisons» n'expliquent pas grand-chose. Les mêmes remarques peuvent s'appliquer au pillage des œuvres d'art qui n'engageait directement aucune extermination 
physique et fut plus restreint dans ses volumes que celui des appartements. Pourtant il a été étudié en détail et a donné lieu à colloques, expositions et publications ${ }^{6}$. Le nom de Rose Valland, résistante sur le «front de l'art », est célèbre ${ }^{7}$. De même, et à l'opposé, au sein de la capitale, de multiples plaques indiquent ici la fusillade d'un résistant, là l'arrestation d'un autre ou encore celle de Juifs finalement déportés (Barcellini et Wieviorka 1995). Aujourd'hui toutes les écoles de la capitale affichent la liste des enfants qu'elles accueillaient en 1940 et qui ont été déportés. Les communes de province instaurent, les unes après les autres, une signalétique indicatrice de la présence de tout lieu d'internement et résistance à celui-ci sur leurs communes ${ }^{8}$. Sur la façade des bâtiments qui ont abrité ces annexes parisiennes de Drancy, par contre, aucune marque ne figure. Disposées aux alentours du quai de la Gare, aujourd'hui déposées du fait de travaux, seules deux plaques indiquent que quelque chose s'est passé mais dans des termes largement erronés. Il apparaît que ces camps furent « oubliés » ni parce qu'ils étaient une petite pièce du puzzle ni parce qu'ils auraient fait l'objet d'une omerta, d'un refoulement. Le bon accueil institutionnel et médiatique fait à l'ouvrage qui raconte leur histoire est un indice fort du contraire ${ }^{9}$.

La question reste donc posée. Y répondre oblige tout d'abord à considérer la nature toujours mémorielle de «l'oubli ». Puisque la conscience de ce dernier ne naît que lorsqu'il disparait, le comprendre passe en réalité par une appréhension du fonctionnement de la mémoire dont il n'est jamais qu'une forme parmi d'autres. Le travail historien lui-même s'explique toujours lui-même par un rapport au passé. Comme le dit Henry Rousso, « la mémoire savante n'a aucune espèce d'indépendance. D'un côté, elle est partiellement, sinon complètement, influencée par une mémoire dominante, souvent d'origine politique ou idéologique, de l'autre, elle entre en concurrence avec d'autres formes de représentations du passé et ne peut donc s'analyser que comme partie intégrante d'un système de représentations » (Rousso 1991 : 252). Cette dimension est, en l'espèce, d'autant plus présente que, originellement, le travail qui a conduit à l'écriture de l'histoire de ces camps a été entrepris à la demande d'une amicale rassemblant quelques témoins, directs ou indirects, de l'existence de ces camps (Weill 2003 : 9-13). Il convient désormais de remonter le fil de leur mémoire pour comprendre comment celui-ci a pu être durablement perdu et récemment retrouvé.

Ces camps ont longtemps cristallisé ce que, à partir de son étude des religions africaines au Brésil, R. Bastide désigne du terme de «trou de mémoire » : « une forme à la fois vide et pleine, vide puisqu'elle n'arrive point à se combler à l'aide des images de la mémoire collective, pleine cependant puisqu'elle n'est point véritablement absence, néant, ou rien, mais sentiment d'un manque et, sentiment agissant, provocateur d'un effort mnémonique » 
(Bastide 1970 : 95). Après 1945, l'évocation de la mémoire des camps ne se fait d'abord que par le manque et le décalage. Le rappel des camps annexes de Drancy, dans les mémoires publiés par certains des anciens internés ou par des membres de leurs familles, ne donne souvent lieu qu'à une phrase brève et factuelle. Dans les différentes versions de ses souvenirs, Georges Wellers, ayant pourtant séjourné huit mois à Austerlitz, n'y consacre qu'un très bref passage (Wellers 1991: 126). Odette Fabius fait, à cet égard, figure d'exception par la longueur du passage qu'elle leur octroie. En 1980, elle-même déportée en tant que résistante, elle évoque, dans ses Mémoires, le passage de son mari, Robert, par Lévitan :

\footnotetext{
«Les nazis faisaient transiter par "Lévitan" le mobilier et les œuvres d'art, fruits de leurs pillages systématiques des domiciles ou propriétés appartenant à des Juifs. [en août] les responsables du camp décidèrent de déporter directement Robert et d'autres prisonniers, outre-Rhin. Avec plusieurs camarades [...] il fut embarqué pour la gare de l’Est » (Fabius $1980: 215-216)$.
}

Par son récit, elle témoigne bien que quelque chose a eu lieu. Cependant, comme si le système social qui porte la mémoire collective de ces camps ne pouvait être retrouvée, le manque et le vide que son absence crée sont ici comblés par des éléments empruntés aux représentations sociales canoniques du passé, le pillage des œuvres d'art d'un côté, celui de la déportation de l'autre.

\section{PHOTO 1}

Façade du 85-87 rue du Fbg St Martin 75010 Paris, décembre 2005, anciennement Magasin Lévitan

Au début de l'année 2002, encore, il en va de même dans le voisinage du 85-87 rue du Faubourg-Saint-Martin dans le $10^{\mathrm{e}}$ arrondissement. Alors que les nouveaux occupants des lieux ont la vague connaissance qu' « il y avait quelque chose avec des juifs pendant la guerre ", seul le pharmacien, dont la vitrine offre un cadre parfait à la façade du bâtiment, sait. Il dit qu'il sait que pendant la guerre, là, il y avait des Juifs, des femmes et des hommes, en attente d'être déportés depuis la gare de l'Est vers les camps d'extermination. Il dit qu'il sait que ces Juifs travaillaient au stockage des œuvres d'art qui, elles aussi, partaient de la gare de l'Est pour l'Allemagne ${ }^{10}$. À la question de savoir comment il a connaissance de ce fait, il répond qu'il le tient de plusieurs clientes. Arrivé récemment dans le quartier, précisant qu'il est lui-même juif, il veut « garder cette mémoire ». Ici le processus d'identification au groupe apparaît pour la première fois. 
Répondant aux avis de recherche diffusés dans les halls des immeubles avoisinants, deux voisines savent elles aussi. L'une habitait derrière, l'autre en face. De ces deux points de vue opposés, elles font pourtant le même récit. Elles gardent l'image de ces Juifs tournant en rond sur le toit, surveillés par des Allemands, en attendant « d'être mis dans des wagons pour partir en déportation, depuis la gare de l'Est $»^{11}$. L' « oubli » de Lévitan n'est pas total. Mais, même conservée, la mémoire de son existence passe par un vide que viennent remplir les canons des représentations du passé. Le lien avec la spoliation économique est maintenu à travers le rappel de l'image dominante du pillage des œuvres d'art ; le lien avec la persécution physique est retenu via la référence à la déportation et l'extermination. À la fois vide et plein, le trou de mémoire des camps annexes de Drancy dans Paris prend corps entre ces deux pôles d'une « mémoire » diffuse.

Comme le précise M. Halbwachs, il ne s'agit cependant là de mémoire que par métaphore :

« C'est là une mémoire empruntée et qui n’est pas la mienne. Dans la pensée nationale, ces événements ont laissé une trace profonde. [...] Pour moi, ce sont des notions, des symboles ; ils se représentent à moi sous une forme plus ou moins populaire; je peux les imaginer; il m'est bien impossible de m'en souvenir » (Halbwachs 1997 : 99).

Ces représentations du passé, empruntées à l'histoire, s'imposent aux acteurs selon un mécanisme proche de celui de bricolage mis en évidence par R. Bastide ${ }^{12}$ :

«chercher ailleurs de nouvelles images pour boucher les trous ouverts dans la trame du scénario et lui redonner ainsi cette signification qui vient non de l'addition de simples éléments, mais de la manière dont ils sont organisés » (Bastide $1970: 96)$.

Devant l'absence de constitution d'une mémoire collective, le scénario est écrit par des figures empruntées à l'histoire. Ce mécanisme est l'indice d'une rupture structurelle entre le groupe des internés d'alors et les groupes sociaux de la société d'après-guerre. Le constat de l'existence d'un trou de mémoire est le premier indice que les processus mémoriels doivent être rapportés à la morphologie sociale et à son évolution. En d'autres termes, il doit conduire à une mise en relation des caractéristiques sociales de ceux qui se souviennent (ou qui oublient) avec celles de ceux qui furent effectivement internés, l'existence d'un trou de mémoire laissant présager que cette mise en parallèle fasse apparaître une situation de rupture et de décalage. 
Cette intuition est d'autant plus forte qu'un processus comparable se retrouve dans le cas du quai de la Gare. Il s'appuie cependant cette fois-ci sur un support physique. Malgré les multiples altérations du paysage (Langlois 1996), un indice du passé a longtemps été conservé. Deux plaques ont été apposées après la guerre sur le site du camp.

\section{PHOTO 2 \\ PHOTO 3}

Photographie sur le site du Quai de la Gare, Antoine Perreaux-Forest.

Les inscriptions qu'elles portent ne décrivent que très partiellement la réalité. Les détenus des camps parisiens ne furent pas « des milliers ». La référence "à la résistance française et aux vaillants cheminots » doit faire avec emphase allusion à l'intervention, à Bobigny, du chef de gare pour éviter la formation du dernier convoi régulier, voulu par Aloïs Brunner, le 17 août 1944 (Klarsfeld 2001 : 1894). Mais cette action a eu lieu près de Drancy non au quai de la Gare. Enfin nulle mention n'est faite de la principale raison d'être de ces camps et des tonnes d'objets et de meubles qui y sont passés. Ici le canon de la déportation physique se retrouve pour boucher le trou de mémoire. Celui de l'engagement résistant remplace par contre la référence au pillage des œuvres d'art. Cependant le mécanisme d'emprunt aux figures structurantes de l'histoire vulgarisée est strictement identique.

En 1993, un événement semble indiquer un premier «réveil» de la mémoire. Démarche inédite, trois anciennes internées dans chacun des trois camps rédigent de brefs témoignages publiés dans le Monde Juif et introduits par une courte présentation ${ }^{13}$. Toutefois, la publication reprend elle-même les plaques évoquées, sans corriger leurs erreurs, et propose des photographies du camp qui ne correspondent absolument pas aux faits. Cette initiative initiale n'a pas de suite. Les auteurs des témoignages n'entretiennent pas de relation durable et n'entament aucune autre action.

Les images alors reproduites montrent le bâtiment des entrepôts frigorifiques comme étant celui de l'internement. Depuis 1971, ces derniers abritent un groupe d'artistes indépendants. Parmi eux, au milieu des années 1990, une femme et deux hommes s'intéressent à ces plaques. L'un a eu son oncle déporté à Auschwitz depuis Drancy. L'autre s'interroge sur les origines juives de sa grand-mère. Au milieu des années 1990, ils décident de faire des recherches sur ce qui s'est passé sur ces lieux. Rapidement, ils veulent faire savoir qu'un « camp de Juifs » a existé dans le quartier. À tort, ils situent d'abord le camp dans l'un des deux seuls bâtiments encore debout, le leur. 
Ils s'adressent alors à la Semapa qui aurait dû, à leurs yeux, prendre en charge ce passé que les travaux de la $\mathrm{Zac}^{14}$ rive gauche, dont elle a la responsabilité, avaient définitivement englouti. Le manque d'écho qu'ils rencontrent se greffe au conflit existant entre les artistes et leur propriétaire désireux de mettre fin à cette expérience créatrice peu rentable. Ainsi, pour ces premiers protagonistes, le silence sur les camps s'explique par une volonté délibérée de cacher et une manipulation politique. Pensant le faire voler en éclats, ils en parlent à la presse (Smoltczyk et Weiss 1997), laissant entendre que le camp pourrait aussi se situer sous la « très grande bibliothèque ». Ils décident de faire appel aux témoins. En publiant une annonce iconoclaste dans un grand quotidien national du soir, ils interpellent des anciens internés. Lorsque l'un des journalistes est l'arrière-petit-fils d'un interné, les deux démarches se rejoignent (Weill 1997).

\section{Cadres sociaux du passé, cadres sociaux de la mémoire}

Individuellement ou par petits groupes, à l'image de celui des trois personnes à l'origine de la publication de 1993, plusieurs répondent à l'annonce. Certains parlent de ce passé dans leur réseau d'interconnaissance et découvrent d'autres «anciens». Puis plusieurs appels sont diffusés dans la presse nationale comme dans certaines revues associatives. Un processus est lancé. Au début de 1998, une association loi 1901 est formée. Baptisée « Amicale AusterlitzLévitan-Bassano », elle a pour objectif de « retracer l'histoire des camps d'internement de travail et de transit parisiens et d'en conserver la mémoire ${ }^{15}$. En creux, l'étude de la formation et de la composition de cette amicale permet de saisir le fonctionnement de la mémoire. Comprendre comment certains veulent et peuvent se souvenir éclaire sur ce qui fait que d'autres, notamment aux positions et identifications sociales différentes, ont longtemps « oublié » et, dans leur majorité, continuent à le faire.

$\mathrm{Au} 1^{\mathrm{er}}$ octobre 2001, avant le début de notre enquête historique, cette amicale compte 29 personnes, au titre de leur ancien internement, de celui de leurs parents et aïeuls ou d'un simple intérêt historique ${ }^{16}$. Cela appelle une première remarque. Alors que, ayant compté certes un petit effectif, les camps parisiens ont vu au moins 629 personnes leur survivre, ceuxci pouvant en parler à leur famille, le taux d'adhésion et d'identification est particulièrement faible.

Plus largement, intégrant des descendants, la composition de cet effectif de 29 personnes peut être établie selon deux focales. La première concerne les caractéristiques sociales de ceux et celles que les enfants ou petits-enfants représentent. La seconde considère ces derniers pour 
eux-mêmes. Mais les deux tiennent compte tant de l'identification extérieure que de celle subjective, bien sûr toujours exprimée dans des mesures variables, qui a pu être appréhendée lors des entretiens menés avec les membres de l'amicale ${ }^{17}$. Aucune des personnes classées comme «issue de mariage mixte» ne se revendique, par exemple, comme «appartenant totalement au judaïsme ».

\section{Composition de l'amicale Austerlitz-Lévitan-Bassano}

Premier mode de calcul : les adhérents comptent pour ceux qu'ils représentent

\begin{tabular}{|l|c|c|c|c|c|c|}
\hline & $\begin{array}{c}\text { Appartenance } \\
\text { «stricte } \\
\text { judaïsme }\end{array}$ & $\begin{array}{c}\text { Issu(e) de } \\
\text { mariage mixte }\end{array}$ & $\begin{array}{c}\text { Marié(e) à un } \\
\text { non-Juif }\end{array}$ & $\begin{array}{c}\text { Femme de } \\
\text { prisonnier }\end{array}$ & $\begin{array}{c}\text { Sans } \\
\text { lien/ } \\
\text { judaïsme }\end{array}$ & $\begin{array}{c}\text { Total } \\
\text { Descendants }\end{array}$ \\
\hline Témoins & 7 & 3 & 3 & 1 & 8 \\
\hline Liés aux \\
lieux
\end{tabular}

Second mode de calcul : les adhérents comptent pour eux-mêmes

\begin{tabular}{|c|c|c|c|c|c|c|}
\hline & $\begin{array}{l}\text { Appartenance } \\
\text { « stricte » au } \\
\text { judaïsme }\end{array}$ & $\begin{array}{c}\text { Issu(e) de } \\
\text { mariage mixte }\end{array}$ & $\begin{array}{c}\text { Marié(e) à un } \\
\text { non-Juif }\end{array}$ & $\begin{array}{l}\text { Femme de } \\
\text { prisonnier }\end{array}$ & $\begin{array}{l}\text { Sans lien / } \\
\text { judaïsme }\end{array}$ & Total \\
\hline Decendants & 6 & 2 & & & & 8 \\
\hline Témoins & 7 & 3 & 2 & 1 & & 13 \\
\hline $\begin{array}{l}\text { Liés aux } \\
\text { lieux }\end{array}$ & 2 & 1 & & & & 3 \\
\hline Historiens & 4 & & & & 1 & 5 \\
\hline Total & 19 & 6 & 2 & 1 & 1 & 29 \\
\hline
\end{tabular}

\section{Encadre 2 : «Classification des internés : listes, déportations et inspections »}

La répartition des internés selon les catégories officielles est difficile. Son retour à Drancy étant ordonné en juin 1944, le groupe des femmes de prisonniers de guerre peut être, lui, clairement identifié. Il compte 64 membres $^{18}$, rapidement rassemblés à Lévitan. Avec certaines de leurs camarades de même statut, celles restées à Drancy, ces femmes sont toutes 
déportées à Bergen-Belsen en juillet $1944^{19}$. L'identification est plus complexe pour les trois autres catégories. Les diverses listes ponctuelles ${ }^{20}$ retrouvées libellées explicitement des appellations statutaires ou de celles, plus rares, de « Mischlinge » ou de «Quart de Juifs » fournissent un premier type d'informations. 73 personnes ont été identifiées comme étant mariées à des non-Juifs, 63 comme étant, au sens large, issues de mariages, ou de familles, mixtes. Cependant cette information est extrêmement partielle et n'est pas à l'abri de fausses déclarations. Elle peut simplement donner une idée des proportions respectives entre le deuxième et le troisième groupe. Mais elle ne permet ni de déterminer leurs effectifs d'ensemble ni celui de la quatrième catégorie. Face à cette impasse, seule l'étude du processus qui a conduit à la déportation de certains, les 30 juin et 31 juillet 1944, permet $d$ 'aboutir à une conclusion solide, tout en étant, en la matière, toujours temporaire ${ }^{21}$.

Aux alentours du 25 juin, l'ensemble des internés des trois lieux est ramené par étapes à Drancy. Alors qu'après le débarquement le remplissage des convois de déportation s'avère plus difficile, Aloïs Brunner est à la recherche de détenus « déportables ». Il procède ainsi au contrôle systématique des papiers. Si là encore l'arbitraire a pu régner, les témoignages recueillis comme les sources écrites retrouvées convergent pour indiquer que ceux qui jusquelà avaient bénéficié d'une dérogation ou d'une simple déclaration sont, dans leur majorité, gardés à Drancy et déportés par les convois 76 et 77, du 30 juin et du 31 juillet 1944. Ils sont 49 au total. Dès lors que l'objectif est celui de la détermination d'un ordre de grandeur ${ }^{22}$, il est possible de considérer l'effectif de ces deux vagues de déportations vers Auschwitz comme un indicateur de celui des personnes passées par les camps annexes mais ne relevant pas dans les faits des statuts officiels. Cela donne une image de la répartition de la population des camps annexes de Drancy.

Les deux calculs sont sensiblement convergents dans les tendances qu'ils indiquent. Les personnes appartenant au judaïsme et s'y identifiant subjectivement sont largement majoritaires. Or, mise en parallèle avec celle établie pour les internés des camps, cette sociographie de l'association frappe par le décalage dans lequel elle s'inscrit. En effet, les internés des camps annexes étaient répartis en quatre groupes principaux : les femmes juives de prisonniers de guerre juifs, les « conjoints d'aryens », les « demi-Juifs » et des détenus juifs que l'on peut qualifier de «classiques» du camp de Drancy. Du fait de la possession d'une compétence particulière, de la détention de vrai/faux « certificats de non-appartenance à la race juive » ou d'une simple déclaration, longtemps suffisante, certains parmi ces derniers pouvaient être affectés à Paris. Il apparaît que seuls $15 \%$ de leurs habitants ont une identité, 
au moins prescrite, totalement juive. À l'inverse, $85 \%$ entretiennent des liens directs et objectifs, d'ordre matrimonial ou généalogique, avec des non-Juifs.

\section{Répartition catégorielle des internés successifs ayant séjourné dans les camps parisiens}

\begin{tabular}{|c|c|c|c|c|}
\hline & $\begin{array}{c}\text { Femmes juives de } \\
\text { prisonniers de guerre } \\
\text { juifs }\end{array}$ & $\begin{array}{c}\text { Mariés à une } \\
\text { personne } \\
\text { non-juive }\end{array}$ & $\begin{array}{c}\text { Issus de mariages } \\
\text { mixtes }\end{array}$ & $\begin{array}{c}\text { Appartenance } \\
\text { prescrite stricte au } \\
\text { judaïsme }\end{array}$ \\
\hline $\begin{array}{c}\text { Proportion } \\
\text { par rapport } \\
\text { au total des } \\
\text { internés }\end{array}$ & $8 \%$ & $46 \%$ & $39 \%$ & $7 \%$ \\
\hline $\begin{array}{c}\text { Nombre } \\
\text { d'internés } \\
\text { par catégorie }\end{array}$ & 64 & 366 & 310 & 55 \\
\hline
\end{tabular}

Cependant, comme le rappellent les études actuelles en sociologie de la religion (Azria 1996 ; Cohen 2000), l'identification objective des personnes, et ce d'autant plus lorsqu'elle reprend, même partiellement, les catégories nazies, ne correspond bien sûr pas forcément à l'autodéfinition que les acteurs donnent d'eux-mêmes. Les données quantitatives sont confirmées par une approche plus qualitative. Fournissant un indice de la culture et des références, en quelque sorte, de ces microsociétés ${ }^{23}$, plusieurs éléments convergent pour dresser un portrait de détachement à l'égard des divers traits d'identification subjective au judaïsme. Tout d'abord, les différentes sources font apparaître que plusieurs des détenus étaient convertis au catholicisme. Plus largement, les jeux de courriers conservés ne font jamais référence à la célébration d'une quelconque fête religieuse juive et n'emploient pas de vocabulaire culturel juif. Ils ne contiennent aucune recommandation en la matière, aucun échange relatif à des éléments culturels ou cultuels juifs. De même, imprimé au quai de la Gare en mars 1944, le journal Camp Camp se présente sous la forme d'un canard satyrique parisien traditionnel où la dérision omniprésente ne fait aucune place à l'humour juif ${ }^{24}$. Enfin, toujours tournés vers le rire, les 17 numéros qui composent le spectacle d'avril 1944 ne comportent ni musiques ni textes issus d'une quelconque tradition juive ${ }^{25}$. S'intitulant « espérance juive », seule une scénette contient un terme issu d'un vocabulaire en lien avec une quelconque forme de judaïsme. Mais ce titre désigne en réalité une performance de 
culturisme, marquant en creux le total décalage qui existe entre le titre et le contenu. Le constat est le même à la lecture des pièces de théâtre qui furent jouées dans le camp ${ }^{26}$.

\section{Mémoire et milieux collectifs}

Ainsi la mise en parallèle des sociographies respectives de l'amicale et des internés frappe par le renversement qu'elle donne à voir. $\mathrm{Si}$, certes variable en valeur, la marginalité des femmes de prisonnier se retrouve dans les deux cas, le rapport entre les proportions des effectifs respectifs relevant des deux autres grands groupes est totalement inversé. Alors que les individus hors statuts, et donc en lien de filiation et d'alliance exclusivement interne au groupe juif, comptaient en tout au plus $15 \%$ des internés, et furent surreprésentés parmi les déportés d'où un fort taux de mortalité, ils forment $65 \%$ des membres de l'association. Tandis que, à l'inverse, constituant à l'époque $85 \%$ des internés, ceux en liens matrimoniaux et généalogiques avec des non-Juifs en composent seulement $35 \%$.

Plus qualitatif, l'ensemble des données concernant l'amicale vient à l'appui de ce constat. Tout d'abord, sa secrétaire générale qui, dans les faits, la dirige, a deux parents juifs. Aujourd'hui veuve, elle fut mariée à un juif. Son grand-père interné à Austerlitz avait obtenu un vrai/faux certificat d'aryanité de son épouse. Pendant la guerre, elle fut membre des Éclaireurs israélites. Elle a de multiples activités et contacts «communautaires ». Elle est insérée dans un réseau d'interconnaissance constitué dans une grande partie de Juifs.

Ensuite, l'observation participante aux assemblées générales de l'amicale confirme la position marginale en son sein des personnes aux identités complexes qui occupaient pourtant la position centrale au sein des camps parisiens. Le 26 mai 2002, quatre véritables prises de parole ont animé la réunion. Toutes émanaient de personnes à l'identité juive, a priori, plus « simple ». Deux d'entre elles émanaient d'anciens déportés, l'une du fils d'une femme de prisonnier déportée à Bergen-Belsen, l'autre de la secrétaire de l'association.

Appréhendé dans toutes ses dimensions, ce décalage entre sociographie de l'amicale et sociographie des internés des camps est un indice fort des modalités du souvenir et donc, en creux, de celles de l'oubli. Lorsque, serait-ce modestement, le passé des camps annexes est remémoré et dit publiquement, cette mémoire est principalement portée par ceux qui y ont tenu un rôle minoritaire mais qui, par contre, peuvent s'identifier plus directement comme appartenant à un même groupe. À l'inverse, concernant une période où les faits et nombre des représentations partagées ont tendance à mettre, séparément, les Juifs d'un côté, les non-Juifs de l'autre, l'appartenance de la majorité des détenus, plus encore de leurs descendants, à la 
fois aux deux ensembles, comme l'identification complexe, à la fois subjective et objective, qui l'accompagne, expliquent ainsi le long trou de mémoire qui entoure le souvenir des camps parisiens.

L'étude des exceptions confirme l'analyse. D'un côté, l'«oubli», la faiblesse de la remémoration, résulte de l'isolement social et d'une position marginale ou de rupture par rapport au groupe ici pertinent dans la structuration de la mémoire. De l'autre, et à l'opposé, il peut également découler d'une insertion sociale forte à un groupe, mais décalée.

Dans le premier cas, le corpus constitué illustre l'approche de M. Halbwachs :

«La succession de souvenirs, même de ceux qui sont les plus personnels, s'explique toujours par les changements qui se produisent dans nos rapports avec les divers milieux collectifs, c'est-à-dire, en définitive, par les transformations de ces milieux, chacun pris à part, et de leur ensemble» (Halbwachs 1997 : 95).

Seul un « changement » dans ses « rapports avec les divers milieux collectifs », une mise en relation, une rencontre suscite chez le témoin, jusque-là uniquement potentiel, l'expression du souvenir.

Les propos de la fille d'un premier mariage d'un ancien d'Austerlitz, comme «conjoint d'aryen », révèlent les difficultés à se remémorer avec d'autres alors qu'on est en fait socialement isolé. Interrogée sur les discussions familiales d'après-guerre, cette femme nous explique :

«On ne peut pas dire qu'on ait eu des gens déportés ou alors de très vieilles connaissances. On était un peu comme des naufragés sur une île par rapport à ce qui se passait en dehors. Bon! il faut dire une chose. Nous étions complètement athées. Neutres depuis toujours. Et donc, les enfants de ma soeur se sont mariés avec des catholiques. Donc il ne restait de Juifs que ma soeur et moi. On est rentré chez nous et puis on ne s'est pas occupé du reste. Et le hasard a fait, il n'y a pas très longtemps, que j'ai une amie qui est une amie de Denise Weill. Elle m'a mise en relation ${ }^{27}$. »

Le silence apparaît ici directement lié à l'isolement, à l'absence de relations avec «des déportés », implicitement «des Juifs », soit, en d'autres termes, ceux de Halbwachs, avec « des milieux collectifs » susceptibles de porter cette mémoire. Inversement la concomitance entre la «mise en relation» avec d'autres - la secrétaire générale de l'amicale, par l'intermédiaire d'une relation - et l'expression du souvenir fait clairement écho au rôle des « faits de communication » dans les mécanismes de remémoration, mis en évidence par Marc 
Bloch (1925). Le souvenir, phénomène social, ne se forme qu'à travers les liens complémentaires entre les membres d'un groupe, porteurs d'un passé commun. "On ne se souvient qu'à condition de se placer au point de vue d'un ou de plusieurs groupes et de se replacer dans un ou plusieurs courants de pensée collective» (Halbwachs 1997 : 65). La reprise de contacts, la mise en relation permet de s'insérer à nouveau dans ces «courants de pensée collective ». Celle-ci est cependant plus improbable quand la position sociale est située à la marge du groupe ou est tellement complexe, faite de nombre d'appartenances distinctes, que l'individu ne peut procéder à une identification minimale.

Un autre exemple donne à voir le rapport direct entre modification des rapports aux milieux collectifs et formation de la mémoire. Lorsque cet ancien du quai de la Gare, interné comme « conjoint d'aryen », nous reçoit pour faire, pour la première fois depuis la guerre, le récit de son internement, il évoque, immédiatement et spontanément, la modification de ses rapports aux « milieux collectifs » relative au récent décès de sa femme :

«Elle n'était pas juive, explique-t-il. Bon, elle ne voulait d'ailleurs pas entendre parler de cette histoire. Elle m'en voulait en fait de m'être, selon elle, laissé arrêter trop facilement. D'ailleurs personne parmi mes enfants ne s'intéresse à cette histoire. Bon, puis il faut savoir que ma femme, comme moi, étions athées militants. »

La rupture totale de cette personne d'avec le groupe social dont il partageait un passé commun, ici pertinent - celui des Juifs avant et sous l'Occupation - explique son long silence. Inversement, c'est sous le signe d'un changement récent de sa relation potentielle à ce milieu - le décès de sa femme non-juive ne souhaitant pas parler de ce passé - que cet homme place immédiatement son témoignage.

Si elle permit qu'il accepte de témoigner, cette transformation des relations avec les différents milieux collectifs reste cependant marginale. Cet homme ne s'investit pas dans l'association. Pour accepter de nous rencontrer, il nous avait, avant toute chose, demandé au téléphone de l'assurer que l'amicale n'avait bien « aucun caractère confessionnel». Afin d'accepter de témoigner d'un des maillons de la persécution des Juifs, cet homme préfère un cadre non lié à l'identité juive. Les liens entre milieux collectifs et mémoire expliquent que les témoins potentiels ne se soient que très peu exprimés et que leurs souvenirs n'aient pas constitué une véritable mémoire collective. Plusieurs anciens internés, comme leur famille, ne parlent pas de ce passé pas tant parce que celui-ci « ne passe pas » que parce qu'eux-mêmes ne trouvent pas leur(s) place(s) par rapport à lui ${ }^{28}$. 
Tout en mettant en évidence le même lien entre mémoire et identification collective, le silence des femmes de prisonnier de guerre passées par les annexes de Drancy peut, lui, être rapporté, à l'inverse, à un très fort sentiment d'appartenance à un groupe structuré mais au souvenir décalé. Juives mariées à des Juifs, ces femmes occupent une position sociale a priori moins marginale au sein des milieux collectifs qui semblent ici déterminants pour la formation de la mémoire. Elles auraient pu fournir un cadre fort pour l'expression d'un souvenir des camps parisiens. Elles ont témoigné mais d'une autre expérience, qui les a toutes rassemblées : leur déportation à Bergen-Belsen. Ayant séjourné ensemble dans des chambrées à Drancy, à Lévitan pour celles transférées à Paris, parties par deux seuls convois, rassemblées dans un camp spécifique en raison de leurs «statuts », elles forment un groupe au sens fort, ayant eu une socialisation, de plusieurs années parfois, qui ne se résume pas au seul internement concentrationnaire (Doerry 2004). Les survivantes sont aujourd'hui encore rassemblées en une amicale. Cette dernière, comme nombre de ses membres, connaît l'existence de celle qui traite des camps parisiens mais aucune démarche n'a été entreprise de sa part pour nourrir de son expérience et des souvenirs accumulés les initiatives de celle-ci.

Contactée, l'une de ces femmes a pensé qu'il n'était pas utile de nous rencontrer. Elle s'en explique :

« [À Lévitan] beaucoup de choses malheureuses ont dû se passer, mais pour ma part, il est resté pour moi un passage transitoire entre Drancy et Bergen-Belsen [...] J'ai tellement l'impression que mon témoignage n'apportera rien de nouveau [...] Je ne me souviens pas de l'intendance de l'organisation. Il devait bien y en avoir une mais elle ne nous pesait pas, à part l'enfermement. J'ai retenu une halte entre deux épreuves cruelles : Drancy et Bergen-Belsen. Mes deux camarades [...], devenues des amies chères, et moi avons toujours pensé que ces quelques mois qui nous rapprochaient de la fin de la guerre nous ont sûrement aidées à survivre. [...] Pardonnez-moi de ne pas vous donner davantage de détails matériels. J'en ai oubliés beaucoup pour ne retenir que cette impression d'entre-deux non pas que nous n'étions pas inquiets, non pas que les endroits n'étaient pas tristes et gris et que nous y étions prisonniers ${ }^{29}$.»

Nous n'avons pu rencontrer aucune de ces femmes porteuses d'une mémoire collective constituée. Situé dans un à-côté, le souvenir des camps annexes n'est, pour elles, que cet « entre-deux », entre Drancy et Bergen-Belsen. Il est ce vide, en d'autres termes, ce trou de mémoire qui marque l'absence d'une structure sociale reconstituée. La situation est en quelque sorte ici inversée. À l'opposé du cas précédent, ces femmes ne se « souviennent » pas non parce qu'elles ne trouvent pas leur place mais parce qu'elles en occupent une fortement définie mais sensiblement décalée. 


\section{Témoignages historiques, récits biographiques}

Cette femme conclut sa lettre en ces termes :

« Il est difficile de parler ainsi de ces mois passés là, sans horreur quand tant d'horreurs se passaient ailleurs que nous avons subies ensuite en Allemagne. »

Finalement restés à l'écart des déportations, dans des conditions matérielles meilleures que celles de Drancy, sans parler de Bergen-Belsen ou d'Auschwitz, nombre des internés des camps parisiens peuvent apparaître comme des «épargnés », du fait d'un statut particulier. Leur éventuel désir de prendre la parole se heurte à l'importance structurante de la figure de la déportation, politique puis raciale, au sein de «l'espace du dicible ${ }^{30}$ de l'expérience de la guerre en France (Wieviorka 1995). Exprimée par les intéressés sous la forme d'une volonté de discrétion liée à cette position relativement "protégée ", la position là aussi marginale occupée par la majorité des anciens internés des camps parisiens à l'égard du passé de la déportation constitue un deuxième facteur explicatif des balbutiements du souvenir des camps parisiens. À nouveau, le lien entre milieux collectifs et expression du souvenir apparaît central.

Dès la Libération, plusieurs internés ont pourtant raconté ce qu'ils avaient vu. Il en est ainsi, en septembre 1944, de Georges Geissmann, dans L'Homme libre, organe de l'Union démocratique socialiste de la Résistance, et de Muriel Schatzmann dans Fraternité. Cette évocation publique a cessé avec le retour des déportés et la prise de conscience de ce qu'avaient été les camps de concentration et d'extermination. À partir d'avril 1945, il ne fut plus question des annexes de Drancy ni sur la scène publique ni dans les publications des milieux de mémoire. Après le retour des déportés, il ne semble pas avoir été légitime pour ceux de ses anciens internés, finalement libérés le 18 août 1944, de raconter leur histoire. Cet homme à qui nous demandions s'il en avait parlé après la Libération, en privé ou en public, nous a répondu :

«Bon! les déportés c'est tout simple, si j'ose dire, ils n'avaient plus envie de parler. Ils pensaient qu'on ne les croirait pas. Donc, moi, j'ai eu pudeur à parler de mon cas [...] Aller au quai de la Gare représentait un havre d'espoir. Évidemment quand on sait ce qui s'est passé avec les déportés, c'était un privilège ${ }^{31}$. » 
Un de ses anciens camarades a exprimé la même impression :

«J'ose le dire ce n'était pas dramatique sauf la séparation. Je m'excuse de dire ça, mais compte tenu d'Auschwitz, c'est le jour et la nuit ${ }^{32}$.»

Mêlée à un sentiment diffus de culpabilité d'avoir échappé à la déportation au prix d'un travail forcé dans le cadre de la spoliation des biens des Juifs, se manifestant notamment par l'accent systématique et hyperbolique mis sur leurs actes de "sabotage » dans leur récit, l'expérience historique marginale vis-à-vis du canon de la déportation de la majorité des internés les a conduits parfois à restreindre l'expression de leur souvenir au cercle familial ${ }^{33}$, le plus souvent à se taire. Marginaux vis-à-vis de l'identification au judaïsme, marginaux visà-vis du groupe des déportés, la majorité des anciens détenus de Lévitan, Austerlitz ou Bassano n'ont pas trouvé des cadres sociaux propices à l'expression de leurs souvenirs.

Il est d'ailleurs apparu que seul le passage par Drancy, camp de transit pour la déportation, pouvait s'inscrire dans des cadres dicibles. Un entretien réalisé avec un ancien d'Austerlitz fut à cet égard révélateur. Venue le voir expressément au sujet des camps annexes, qu'il voulait ardemment faire connaître, plus des deux tiers de l'entretien ont pourtant porté sur Drancy. Alors que nous parlions depuis quelques minutes et sans équivoque, du quai de la Gare, nous lui demandâmes : «Pouvez-vous dessiner le camp ?». «Oui je peux essayer ». Prenant un stylo, il commença à en poser les contours : « Des bâtiments ici, là et ici. Bon il y avait trois ailes, comme un U vous voyez...»

\section{PHOTO 4}

Dessin de Monsieur Roger M., réalisé le 8 avril 2002.

\section{PHOTO 5}

Photographie du camp de Drancy, CCXLV_280 B, Mémorial de la Shoah/CDJC.

Le camp de Drancy apparut peu à peu sur la feuille. Surprise, nous demandâmes : « Ah, bon ! donc cela était conçu comme Drancy? ». «Oui c'est Drancy, c'est ça (silence) Ah ! vous parliez du quai de la Gare ?» ${ }^{34}$ Comme la réalité dont il témoigne, le souvenir des camps parisiens occupe, à son tour, une position annexe vis-à-vis de la mémoire de Drancy. Alors que ces cadres sociaux de la mémoire président à l'expression des souvenirs, ils encadrent également le contenu de ces derniers. Encore aujourd'hui, même après la conduite du travail d'histoire orale, ceux qui furent finalement déportés sont toujours les seuls à avoir fait mention d'aspects véritablement douloureux des conditions de vie dans ces camps. 
Déportées pour Auschwitz, le 30 juin 1944, Yvonne Klug évoqua le froid extrême ${ }^{35}$, Vivette Baharlia-Politi décrivit, elle, les énormes rats qui se glissaient la nuit dans son $\operatorname{lit}^{36}$. Les souffrances et les peurs que, à n'en pas douter, les internés, finalement libérés en 1944, ont également éprouvées n'ont jamais été décrites dans les témoignages que nous avons recueillis. Ces récits ne parlent jamais de ceux qui les font mais seulement de ce qu'ils ont vu. L'isolement et la position à la marge ont fait que nous avons principalement récolté des « témoignages historiques 》 sur la spoliation et non des « récits biographiques 》 vécus comme identitaires (Pollak 1990 : 186-201), corollaires de la formation d'un cadre véritablement collectif minimal de la mémoire. L'éclosion, notamment à travers la Commission Mattéoli ${ }^{37}$, de la spoliation comme sujet historique français important a notamment permis cette prise de parole. Selon divers degrés et sous des formes différentes, nos interlocuteurs y font tous référence lors des entretiens. Pour des individus pour qui l'identification, selon un continuum variable, qui accompagne toujours la mémoire, est malaisée, la formation d'un « espace du dicible» public concernant légitimement tous les Français, parmi lesquels ils figurent de manière davantage univoque, permet parfois de mettre fin au silence. Ayant joui d'une couverture médiatique relativement large, la publication du livre a accentué ce processus. Elle a amené quatre nouvelles personnes à vouloir que nous les rencontrions pour nous confier leurs récits.

Enfin, à travers leurs propos, les témoins ont aussi, comme par procuration et à leur manière, témoigné de la déportation, la disparition finale des biens possédés par ceux qui allaient être exterminés les faisant disparaître une seconde fois. Les images et symboles de la déportation de masse furent très présents. Lors des entretiens, le lapsus entre Auschwitz et Austerlitz fut fréquent. Nombreux furent ceux qui, parfois en préambule, souvent de façon principale, racontèrent en détail les déportations de leur famille et celles auxquelles ils avaient assisté lors de leurs divers séjours à Drancy.

Dans cette perspective, la conduite d'une recherche historique universitaire et scientifique offrit un terrain plus «neutre» quant aux espaces d'indentification induits, propice à l'expression du témoignage. Si le processus qui permet ce dernier met en évidence les cadres sociaux de la mémoire, il ne s'agit pas pour autant de l'expression d'une mémoire collective structurée. Comme l'a expliqué M. Halbwachs :

«pour que notre mémoire s'aide de celles des autres, il ne suffit pas que ceux-ci nous apportent leurs témoignages : il faut encore qu'elle n'ait pas cessé de s'accorder avec leurs mémoires et qu'il y ait assez 
de points de contacts entre l'une et les autres pour que le souvenir qu'ils nous rappellent puisse être reconstruit sur un fondement commun » (Halbwachs $1997: 63$ ).

Souvent considérée comme un signe fort de l'existence d'une mémoire collective (Winter et Sivan 1999: 6-40), la mise en place d'une amicale officielle aurait en effet pu révéler ce « fondement commun » puis fournir un cadre à l'expression de cette mémoire collective. Cela ne fut pas le cas. Dès la définition de ses statuts, ses membres n'ont d'ailleurs prévu aucune véritable sociabilité caractéristique des associations de mémoire. Aucune activité ou discussion commune ne fut instaurée. Ce travail de mémoire n'affichait qu'un seul but: commanditer un travail d'histoire. Seule la question de la pose des plaques sur les différents lieux justifie encore son existence. Sa dissolution est prévue dès que cette dernière mission « historique » sera accomplie.

À rebours, la conduite de notre propre recherche scientifique a sensiblement modifié les cadres même de la mémoire en créant un espace du dicible élargi où la parole de certains témoins peut désormais apparaître comme légitime. Elle a ainsi modifié l'identification de certains dans leur rapport au passé. Dans cette perspective, l'écriture de l'histoire finalement faite analyse de la mémoire n'est pas sans conséquence sur le « réveil » même de cette dernière qui permet, à son tour, par un retour aux techniques de l'histoire orale, l'enrichissement du récit du passé lui-même. La boucle est en quelque sorte bouclée.

Dans La mémoire, l'histoire, l'oubli, Paul Ricœur (2000) ouvrait sa propre réflexion sur les rapports de l'histoire et de la mémoire en rappelant que «l'autonomie de la connaissance historique par rapport au phénomène mnémonique demeure la présupposition majeure d'une épistémologie cohérente de l'histoire en tant que discipline scientifique et littéraire ». Certes, mais l'exemple de l'écriture de l'histoire des camps parisiens a aussi montré l'imbrication étroite, et croisée, entre souvenirs individuels, mémoire collective, mémoire empruntée et histoire. L'analyse du trou de mémoire qui les entourait a permis de mettre en évidence le rôle de «l'interpénétration du collectif et de l'individuel» (Lavabre 1994: 17) au cœur des processus mémoriels alors apparus comme des faits sociaux dont il est possible d'identifier les déterminants. La mémoire apparaît soumise à des cadres sociaux. En cela elle est explicable, au moins en partie, comme un fait social, ici appréhendé à travers l'évocation de lieux du passé. Pourtant, par l'approche du fonctionnement du souvenir qu'ils mettent en évidence, ceux-ci différent nettement de ceux distingués par Pierre Nora. Ils ne fondent pas une histoire « au second degré » (Nora 1992 : 25), mais incitent plutôt à développer une sociologie dite « de la mémoire» dont la voie a jusqu'ici était trop peu explorée (Lavabre 1998). Sa 
possibilité devrait inciter historiens, sociologues et politologues à une réflexion peut-être d'avantage empirique sur l'épaisseur et la complexité de l'objet mémoire, la connaissance du passé, elle-même, s'en trouverait renforcée.

Assouline, Pierre. 1997. Le Dernier des Camondo. Paris, Gallimard.

Azoulay, Floriane et Annette Wieviorka. 2000. Le Pillage des appartements et son indemnisation. Paris, Mission d'étude pour la spoliation des juifs de France, La Documentation française.

AZRIA Régine. 1996. "Réidentification communautaire du judaïsme », in Danièle HervieuLéger et Grace Davie (éd.), Identités religieuses en Europe. Paris, La Découverte (Recherches) : 253-267.

Barcellini, Serge et Annette Wieviorka. 1995. "Passant, souviens-toi!». Les lieux du souvenir de la Seconde Guerre mondiale en France, Paris, Plon.

BASTIDE, Roger. 1970. "Mémoire collective et sociologie du bricolage », L'Année sociologique, vol. 21. : pp65-108

Bensimon, Doris et Sergio Della Pergola. 1984. La population juive de Fance : sociodémographie et identité. Jérusalem, Université hébraïque de Jérusalem-Paris, CNRS.

Bloch, Marc. 1925. «Mémoire collective, traditions et coutumes. À propos d'un livre récent », Revue de synthèse historique, $\mathrm{n}^{\circ}$ 118-119:73-83.

Cantonne, Jean-Marie. 2001. Portraits volés. Paris (Livre de Poche).

Chaumont, Jean-Michel. 1997. La Concurrence des victimes. Génocide, identité, reconnaissance. Paris, La Découverte (Textes à l'appui. Série sociologie).

CoHEN, Martine. 2000. « Les Juifs de France. Modernité et identité », Vingtième Siècle. Revue d'histoire, $\mathrm{n}^{\circ} 66: 91-106$.

ConAn, Éric et Henry Rousso. 1996 [1994]. Vichy, un passé qui ne passe pas. Paris, Gallimard.

Confino, Alon. 1997. "Collective Memory and Cultural History: Problems of Method», American Historical Review, $\mathrm{n}^{\circ} 105$ : pp1386-1403.

DOERRY, Janine. 2004. «Juden aus frankreich in Aufenthaltslager Bergen-Belsen», Magisterarbeit, vorgelegt an der Universität Hannover, Historisches Seminar. Durchgesehene Fassung, avril.

Dreyfus, Jean-Marc et Sarah Gensburger. 2003. Des Camps dans Paris. Austerlitz, Lévitan, Bassano, juillet 1943-août 1944. Paris, Fayard (Pour une histoire du XX siècle). 
FABIUS, Odette. 1980. Un lever de soleil sur le Mecklembourg. Mémoires. Albin Michel, Paris.

FERro, Marc. 2002. Les tabous de l'histoire. Paris, Nil éditions.

GENSBURGER, Sarah. 2002. «Les figures du Juste et du Résistant et l'évolution de la mémoire historique française de l'Occupation », Revue française de science politique, $\mathrm{n}^{\circ} 2$ : pp291322.

Gensburger, Sarah et Marie-Claire Lavabre. 2005. «Entre "devoir de mémoire" et "abus de mémoire" : la sociologie de la mémoire comme tierce position », in Bertrand Müller (éd.), Histoire, mémoire et épistémologie. À propos de Paul Ricour. Lausanne, Payot (Sciences humaines).

HalbWachs, Maurice. 1997 [1951]. La mémoire collective. Paris, Albin Michel (Bibliothèque de l'évolution de l'humanité).

HISTORIA. 2004. « Les tabous de 1'histoire », avril, n688 : pp76-77.

KLARSFELD, Serge. 2001 [1993]. Le calendrier de la persécution des Juifs en France 19401944. Paris, Fayard.

Klarsfeld, Serge. 1978. Le Mémorial de la déportation des Juifs de France. Paris, Association Beate et Serge Klarsfeld.

LANGLOIS, Gilles-Antoine. 1996. Le Guide du promeneur $13^{e}$ arrondissement, Paris, Parigramme.

Lavabre, Marie-Claire. 1994. Le Fil rouge. Sociologie de la mémoire communiste. Paris, FNSP.

LAVABre, M.-C. 1998. "Maurice Halbwachs et la sociologie de la mémoire », Raison présente, $\mathrm{n}^{\circ} 128: 47-56$.

Lavabre, M.-C. 2000. "Usage et mésusage de la notion de mémoire », Critique internationale, avril : pp48-57.

Lavabre, M.-C. 2004. «Roger Bastide, lecteur de Maurice Halbwachs » in Deloye, Yves, Haroche, Claudine (dir.) Maurice Halbwachs : espaces, mémoires et psychologie collective, Paris, Publications de la Sorbonne.

LE MONDE JUIF. 1993. $\mathrm{n}^{\circ} 146$.

LENOIR, Frédéric. 2001. « Bricolage néotraditionnel et « bris-collage postmoderne », Archives de Sciences Sociales des Religions, $\mathrm{n}^{\circ} 114$, avril-juin : pp50-54.

Les chefs d'œuvre des collections privées françaises retrouvées en Allemagne par la Commission de récupération artistique et les services alliés. 1946. Paris, ministère de l'Éducation nationale. 
MANUEL, Robert. 1975. Qu'allais-je faire dans cette galère ? Paris, Émile-Paul, 1975.

MARY, André. 2000. Le bricolage africain des héros chrétiens, Paris, Cerf, 2000.

Nicholas, Lynn H. 1994. The rape of Europe: The Fate of Europe's Treasures in the Third Reich and the Second World War. New York, Knopf, (trad. fr. Le Pillage de l'Europe. Les œuvres d'art volées par les nazis. Paris, Seuil, 1995).

NorA, Pierre (éd.). 1992. Les Lieux de mémoire, t. 3, Les France, vol. 3, Paris, Gallimard.

Pollak, Michael. 1990. L'Expérience concentrationnaire. Essai sur le maintien de l'identité sociale. Paris, Métailié, (Collection Leçons de choses).

Pollak, M. 1993. "Mémoire, oubli, silence» in Une Identité blessée, Paris, éditions Métailié, (Collection Leçons de choses).

RAJfus, Maurice. 1996. Drancy, un camp de concentration très ordinaire, 1941-1944. Paris, Le Cherche midi éditeur (Documents).

RAPHAËL, Freddy. 1980. "Le travail de la mémoire et les limites de l'histoire orale», Annales. Histoire, sciences sociales, vol. 35, $\mathrm{n}^{\circ} 1$ :127-145.

Riceeur, Paul. 2000. La mémoire, l'histoire, l'oubli. Paris, Seuil (L'ordre philosophique).

Roseman, Mark. 2002. The Villa, the Lake, the Meeting. Londres, Penguin Press, 2002 (trad. fr. Ordre du jour Génocide le 20 janvier 1942. Paris, Louis Audibert, 2002).

Rousso, Henry. 1990. Le Syndrome de Vichy. Paris, Seuil.

Rousso, H. 1991. " Pour une histoire de la mémoire collective : L'après-Vichy », in Denis Peschanski, Michael Pollak, Henry Rousso, Histoire, politique et sciences sociales, Bruxelles, Complexes.

SmoltczyK, Alexander et Maurice Weiss. 1997. « Die Türme des Schweigens », Die Zeit, 23 janvier.

Stoltzfus, Nathan. 1996. Resistance of the Heart. Intermarriage and the Rosenstrasse Protest in Nazi Germany. New York, Norton.

Ternon, Yves. 1999. Du négationnisme : mémoire et tabou. Paris, Desclée de Brouwer.

THE JERUSALEM REPORT. 2004. « Paris’ Dirty Secret », jeudi 21 octobre.

Todorov, Tzvetan. 1995. Les abus de la mémoire. Paris, Arléa.

WeILl, Denise. 2003. «Avant-propos », in Jean-Marc Dreyfus, Sarah Gensburger, Des Camps dans Paris : Austerlitz, Lévitan, Bassano, juillet 1943-août 1944. Paris, Fayard (Pour une histoire du $\mathrm{Xx}^{\mathrm{e}}$ siècle). 
WeILL, Nicolas. 1997. « La bibliothèque François-Mitterand à l'ombre d'un camp nazi », Le Monde, 23 octobre.

WeILL, N. 2003. Une histoire personnelle de l'antisémitisme. Paris, Robert Laffont.

WELlERs, Georges. 1991. Un juif sous Vichy. Paris, Tirésias.

Wieviorka, Annette. 1995. Déportation et Génocide. Entre la mémoire et l'oubli. Paris, Hachette (Pluriel).

Winter Jay et Emmanuel Sivan. 1999. War and Remembrance in the Twentieth Century. Cambridge, Cambridge University Press, (Studies in the social and cultural history of modern warfare).

1. Sur ce «paradigme de la mémoire stratégique », voir Gensburger 2002 : 291-322.

2. Cette mise en relation des deux auteurs a été d'abord opérée par Marie-Claire Lavabre (1994 et 2004).

3. Mis à part les brèves descriptions faites par Maurice Rajfus (1996: 333-340) et Serge Klarsfeld (2001: 1575). Au sein de la dense littérature sur la spoliation artistique, seul l'ouvrage de Lynn $\mathrm{H}$. Nicholas précise que l'Opération fait finalement appel à des Juifs de Drancy, sans d'autres détails (Nicholas 1994 : 168).

4. Bombardé à la Libération, reconstruit puis détruit, l'emplacement même du lieu a changé d'adresse. Le quai de la Gare est aujourd'hui le quai Panhard-Levassor.

5. Le journaliste d'Historia a, par exemple, voulu nous interroger pour sa rubrique «Les tabous de l'histoire » (Historia 2004 : 76-77).

6. Voir du catalogue d'exposition à l'orangerie des Tuileries, juin-août 1946 (Les chefs d'ceuvre des collections... 1946) au roman policier de Jean-Marie Cantonné (2001).

7. Encore le 27 avril 2005, une plaque au nom de Rose Valland a été inaugurée au Jeu de paume.

8. Par exemple, la ville de Grenoble a mis en place des panneaux au cœur de la ville pour signaler les lieux où les «Résistance juive » a agi dans la commune sous l'Occupation.

9. Le travail de recherche a reçu l'aide financière de la Semapa (Société d'économie mixte d'aménagement de Paris) et de la Fondation pour la mémoire de la Shoah. La sortie des Camps dans Paris qui en est l'aboutissement a donné lieu à un événement organisé par la mairie du $13^{\mathrm{e}}$ arrondissement. De nombreux journaux ont rendu compte du livre.

10. Entretien, 13 mars 2003, Paris. Le bâtiment est tout près de la gare de l'Est.

11. Habitant des étages élevés, elles ont effectivement dû voir la promenade des détenus. Entretiens respectivement les 15 mai et 16 mai 2003, Paris.

12. Sur la discussion de la pertinence du réemploi de ce terme et sur la question de sa pertinence, voir André Mary (2000) et Frédéric Lenoir (2001).

13. La première tentative d'écriture de l'histoire sur cette question prit la forme de trois articles publiés dans Le Monde Juif (1993).

14. Zone d'aménagement concerté.

15. Statuts déposés le 14 janvier 1998 à la préfecture de police de Paris.

16. Nous définissons l'adhésion à l'amicale par le paiement de la cotisation et la présence sur la liste conservée par sa secrétaire générale.

17. Vingt-six entretiens ont été réalisés en 2002 et 2003. 
18. Archives du Beit Lohamei HaGhetaot, Dutch Archive Section BHL, Dossier Josef Weiss, $\mathrm{n}^{\circ} 317$.

19. Les convois des 21 et 24 juillet 1944.

20. Nombre d'entre elles sont conservées dans le rouleau 23 des archives de l'UGIF, CDJC.

21. Les déportations antérieures sont aléatoires. Elles résultent de manquements disciplinaires ou d'évasions. Leur effectif est donc dans un premier temps sorti de l'échantillon pour être ensuite ventilé conformément aux proportions établies de façon générale. Cela représente 53 personnes.

22. Sur cette technique (Bensimon et Della Pergola 1984).

23. Il ne s'agit pas, bien sûr, de prétendre décrire une conscience collective partagée mais de fournir des éléments d'appréhension de l'univers d'identification que représentaient ces camps. Chaque expérience individuelle délimite autant de «micromondes» à la fois imbriqués et distincts (Pollak $1990: 17-18$ ).

24. CDJC, Carton de l'Amicale Austerlitz-Lévitan-Bassano, Journal Camp-Camp, mars 1944.

25. CDJC, CMXVII-13, 5, « Programme Austerlitz Folies », 9 avril 1944.

26. CDJC, UGIF 93, Pièces de théâtre, non datées.

27. Entretien avec $\mathrm{M}^{\mathrm{me}}$ Denise B., 29 mars 2002, Paris.

28. Robert Manuel mentionne simplement sa nature de « sang-mêlé » et passe très rapidement sur son passage à Drancy (Manuel, 1975). Contactée, sa fille n'a pas répondu.

29. Lettre de $\mathbf{M}^{\mathrm{me}}$ Paule L., 4 novembre 2002.

30. L'expression est empruntée à Michael Pollak (1990 : 201).

31. Entretien avec M. Roger M., 8 avril 2002, Paris.

32. Entretien avec M. Jean L., 17 mars 2003, Paris.

33. L'histoire du grand-père de Denise Weill avait été transmise (Weill $2003: 23$ et 185-186).

34. Entretien avec M. Roger M., 8 avril 2002, Paris.

33. Yvonne Klug, Le 8616 revint. Souvenirs d'une rescapés du camp d'extermination d'Auschwitz, 1945, non publié, consulté au CDJC, Carton de l'Amicale Austerlitz-LévitanBassano.

34. Vivette Politi, Mémoires, non publié, consulté au CDJC, Carton de l'Amicale AusterlitzLévitan-Bassano.

37. Commission créée en 1997 à l'initiative d'Alain Juppé, alors Premier ministre, pour enquêter sur la spoliation des Juifs de France sous l'Occupation. 